\title{
EGU21-2892
}

https://doi.org/10.5194/egusphere-egu21-2892

EGU General Assembly 2021

(c) Author(s) 2021. This work is distributed under

the Creative Commons Attribution 4.0 License.

\section{High spatial and temporal resolution L- and C-band Synthetic Aperture Radar data analysis from the yearlong MOSAiC expedition}

\author{
Malin Johansson ${ }^{1}$, Suman Singha ${ }^{2}$, Gunnar Spreen ${ }^{3}$, Stephen Howell ${ }^{4}$, Shin-ichi Sobue ${ }^{5}$, and \\ Malcolm Davidson ${ }^{6}$ \\ 1'Department of Physics and Technology, UiT The Arctic University of Norway, Tromsoe, Norway (malin.johansson@uit.no) \\ ${ }^{2}$ Maritime Safety and Security Laboratory, Remote Sensing Technology Institute (IMF), German Aerospace Center (DLR), \\ Bremen, Germany (human.singha@dlr.de) \\ ${ }^{3}$ Institute of Environmental Physics, University of Bremen, Bremen, Germany (gunnar.spreen@uni-bremen.de) \\ ${ }^{4}$ Climate Processes Section, Science and Technology Branch, Environment and Climate Change Canada / Government of \\ Canada, Toronto, Canada (stephen.howell@canada.ca) \\ ${ }_{5}^{5} J a p a n$ Aerospace Exploration Agency (JAXA), Tsukuba, Ibaraki, Japan (sobue.shinichi@jaxa.jp) \\ ${ }^{6}$ European Space Agency (ESA), Mission Science Division, Noordwijk, the Netherlands (Malcolm.Davidson@esa.int)
}

In the yearlong MOSAIC expedition (2019-2020) R/V Polarstern drifted with sea ice through the Arctic Ocean, with the goal to continually monitor changes in the coupled ocean-ice-atmosphere system throughout the seasons. A substantial amount of synthetic aperture radar (SAR) satellite images overlapping the campaign was collected. Here, we investigate the change in polarimetric features over sea ice from the freeze up to the advanced melt season using fully polarimetric Lband images from the ALOS-2 PALSAR-2 and fully polarimetric C-band images from the RADARSAT-2 satellite SAR sensors.

Three different sea ice types are investigated, young ice, level first year ice and deformed first and second-year ice. Areas of deformed and level sea ice were observed in the vicinity of R/V Polarstern and these areas are included whenever possible in the yearlong time series.

Comparing the different sea ice types, we observe that during the freezing season there is a larger difference in the co-polarization channels between smooth and deformed ice in L-band compared to C-band. Similar to earlier findings we observe larger differences between young ice and deformed ice backscatter values in the L-band data compared to the C-band data. Moreover, throughout the year the HV-backscatter values show larger differences between level and deformed sea ice in L-band than C-band. The L-band data variability is significantly smaller for the level sea ice compared to the deformed sea ice, and this variability was also smaller than that observed for the overlapping C-band data. Thus L-band data could be more suitable to reliable separate deformed from level sea ice areas.

Within the L-band images a noticeable shift towards higher backscatter values in early melt season compared to the freezing season for all polarimetric channels is observed, though no such strong trend is found in the C-band data. The change in backscatter values is first noticeable in the C- 
band images and later followed by a change in the L-band images, probably caused by their different penetration depth and volume scattering sensitivities. This change also results in a smaller backscatter variability.

The polarization difference (PD; $\mathrm{V}$-HH on a linear scale) show a seasonal dependency for the smooth and deformed sea ice within the L-band data, whereas for the C-band data no such trend is observed. For the L-band data were the PD variability for all ice classes reasonably small for the freezing season, with a significant shift towards larger variability during the early melt season, though during this time period the mean PD values remained similar. However, once the temperatures reached above $0^{\circ} \mathrm{C}$ both the variability and the mean values increased significantly.

Overall, our results demonstrate that the C- and L-band data are complementary to one another and that through their slightly different dependencies on season and sea ice types, a combination of the two frequencies can aid improved sea ice classification. The availability of a high spatial and temporal resolution dataset combined with in-situ information ensures that seasonal changes can be fully explored. 